\title{
A Transgenic Mouse Model for Gene-Nutrient Interactions
}

\author{
Jing X. Kang \\ Department of Medicine, Massachusetts General Hospital and Harvard Medical School, Boston, Mass., USA
}

\section{Key Words}

Transgenic mouse modelcGene-nutrient interactions • Caenorhabditis elegans $\cdot$ Polyunsaturated fatty acids

\begin{abstract}
Diets or nutritional supplements contain many nutrients and other components that may interact, which adds a layer of complexity to their evaluation. A well-controlled experimental model that can eliminate or minimize the confounding factors of diet is critical for addressing nutrient-gene interactions. The newly generated fat- 1 transgenic mouse was genetically engineered to carry a gene, namely fat-1, from the round worm Caenorhabditis elegans and is capable of converting $n-6$ to $n-3$ fatty acids (which is naturally impossible in mammals), leading to an increase in $n-3$ fatty acid content with a balanced $n-6 / n-3$ fatty acid ratio in all tissues, independent of diet. Recent studies using this model indicate that balancing the tissue $n-6 / n-3$ ratio could exert a significant effect on gene expression. The fat- 1 mouse model allows carefully controlled studies to be performed in the absence of restricted diets, which can create confounding factors that limit studies of this nature.
\end{abstract}

Copyright $\odot 2008$ S. Karger AG, Basel

\section{Introduction}

Polyunsaturated fatty acids (PUFAs) can be defined as fatty acids of 18 carbons or more in length, containing two or more double bonds. They can be classified into

\section{KARGER}

Fax +41613061234

E-Mail karger@karger.ch

www.karger.com
(C) 2008 S. Karger AG, Basel

Accessible online at: www.karger.com/jnn two groups, $n-6$ or $n-3$, depending on the position (n) of the double bond nearest the methyl end of the fatty acid.

The major n-6 PUFAs are linoleic acid (LA, 18:2n-6) and arachidonic acid (AA, 20:4n-6), and the major $n-3$ fatty acids include $\alpha$-linolenic acid (ALA, 18:3n-3), eicosapentaenoic acid (EPA, 20:5n-3) and docosahexaenoic acid (DHA, 22:6n-3). They are essential nutrients because they cannot be synthesized de novo in the human body as well as in many other animals. Their contents in tissues are, therefore, determined by dietary intake.

These two classes (n-6 and n-3) of essential fatty acids can exert a wide range of biological effects. In addition to being a source of energy production, these fatty acids can act as determinants of the physiochemical properties of cell membranes; as substrates for the production of signaling molecules or functioning mediators, and as modulators in the regulation of gene expression. Therefore, the essential fatty acids can profoundly affect the physiological activity and pathological process through different mechanisms [1]. Although both n-6 and n-3 PUFAs are essential lipids for good health, they are metabolically and functionally distinct and often have important opposing physiological functions. Thus, their balance is important for homeostasis and normal development [1].

The contents of n-6 and n-3 fatty acids in human diet have undergone a dramatic change during the evolution and civilization of human beings [2]. The foods available to our ancestors before were quite different from what we eat today. It is believed that the 'ancient' foods were rich in $n-3$ fatty acids and had a balance between $n-6$ to $n-3$ fatty acids (i.e. $n-6 / n-3$ ratio $\approx 1: 1$ ). Since this balancing

Jing X. Kang, MD, PhD

Massachusetts General Hospita

149 - 13th Street, Room 4433

Charlestown, MA 02129 (USA)

Tel. +1 617726 8509, Fax +1 617726 6144, E-Mail kang.jing@mgh.harvard.edu 
fatty acid profile existed for so long during evolution, the human body established its genetic pattern (what gene is needed or not needed for fatty acid synthesis) based on that circumstance. In the past century the industrial revolution, the emergence of agribusiness with processed foods, grain-fattened livestock, and hydrogenation of vegetable fats have all reduced the content of $n-3$ fatty acids and increased n- 6 fatty acids. Consequently, modern Western diets are deficient in $n-3$ fatty acids, but have too much $n-6$ fatty acids with an $n-6 / n-3$ fatty acid ratio of 15-20:1 [3]. Obviously, this ratio is contradictory to our genetic profile that was established based on a 1:1 ratio.

The shift in the $n-6 / n-3$ fatty acid ratio, especially the deficiency of $\mathrm{n}-3$ fatty acids, might have imposed a risk for modern diseases (e.g. cardiovascular disease, cancer, etc.) and thereby a serious threat to public health $[2,3]$. The tissue status (profile) of these fatty acids (particularly the ratio of n-6 to n-3) and their interaction with gene expression are now a subject of scientific as well as clinical interest. Thus, developing novel animal models that allow evaluation of the importance of the tissue status of these nutrients under a well-defined condition is warranted.

\section{Modification of Essential Fatty Acid Composition by Genetic Approaches}

So far the only possible way to enrich tissues with n-3 fatty acids in mammals has been the dietary provision of n-3 fatty acids, because humans and most animals are unable to synthesize them de novo, nor can they convert n-6 to n-3 fatty acids due to the lack of genes for that feat [1]. Traditionally, researchers feed animals diets supplemented with different fats or oils in order to create different tissue fatty acid profiles for their studies. However, confounding factors of diet seem to be unavoidable in studies with dietary supplementation and have been a big concern for nutritional research [4], particularly for studies examining nutrient-gene interactions.

An approach to eliminating the need of $n-3$ fatty acid supplementation is to produce $\mathrm{n}-3$ fatty acids endogenously, ideally from n-6 fatty acids using genes found in some lower life, such as the fat-1 gene from the roundworm Caenorhabditis elegans. This gene encodes an n-3 fatty acid desaturase that can introduce a double bond into $n-6$ fatty acids at the $n-3$ position of their hydrocarbon chains to form $\mathrm{n}-3$ fatty acids $[5,6]$ (fig. 1 ).

A Transgenic Mouse Model for

Gene-Nutrient Interactions

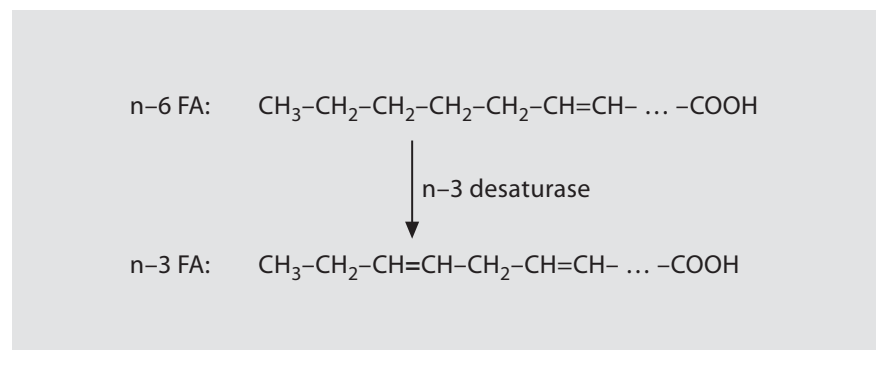

Fig. 1. Conversion of $n-6$ fatty acids (FA) to $n-3$ fatty acids by an $\mathrm{n}-3$ desaturase that does not exist in mammalian cells. The $\mathrm{n}-3$ desaturase can catalyze introduction of a double bond into $n-6$ fatty acids at the $n-3$ position of their hydrocarbon chains to form $\mathrm{n}-3$ fatty acids.

We have used the fat-1 gene to generate a transgenic mouse capable of converting n-6 to n-3 fatty acids [7]. To heterologously express $C$. elegans $n-3$ fatty acid desaturase in mice, we modified the fat- 1 gene encoding this protein by optimization of codon usage for mammalian cells and coupled it to a chicken $\beta$-actin promoter (which allows high-level and broad expression of the transgene in mice). We then microinjected the expression vector into fertilized eggs to produce transgenic mouse lines [7].

Both transgenic and wild-type mice are maintained on a diet high in $\mathrm{n}-6$ fatty acids (mainly LA) with very little $n-3$ fatty acids ( $\sim 0.1 \%$ of total fat supplied). Under this dietary regime, wild-type mice have little or no n-3 fatty acid in their tissues because the animals cannot naturally produce $n-3$ from $n-6$ fatty acids, whereas the fat1 transgenic mice have significant amounts of $n-3$ fatty acids (derived from n-6 fatty acids) in their tissues [7]. Figure 2 shows the differential fatty acid profiles of total lipids extracted from skeletal muscles of age- and sexmatched wild-type and transgenic mice. In the wild-type animals, the PUFAs found in the tissues are mainly (98\%) the n-6 LA (18:n-6) and AA (20:4n-6) with trace (or undetectable) amounts of $\mathrm{n}-3$ fatty acids. In contrast, there are large amounts of n-3 PUFAs, including ALA (18:3n3), EPA (20:5n-3), docosapentaenoic acid (DPA, 22:5n-3) and DHA (22:6n-3), in the tissues of transgenic mice. Accordingly, the levels of the $\mathrm{n}-6$ fatty acids LA and AA in the transgenic tissues are significantly reduced, indicating a conversion of $n-6$ to $n-3$ fatty acids. The resulting ratio of $n-6$ to $n-3$ fatty acids in the tissues of transgenic animals is close to 1 . This $n-3$-rich profile of lipid with a balanced ratio of $n-6$ to $n-3$ and an even more balanced $\mathrm{AA} /(\mathrm{EPA}+\mathrm{DPA}+\mathrm{DHA})$ can be observed in all of the or-

J Nutrigenet Nutrigenomics 2008;1:172-177 


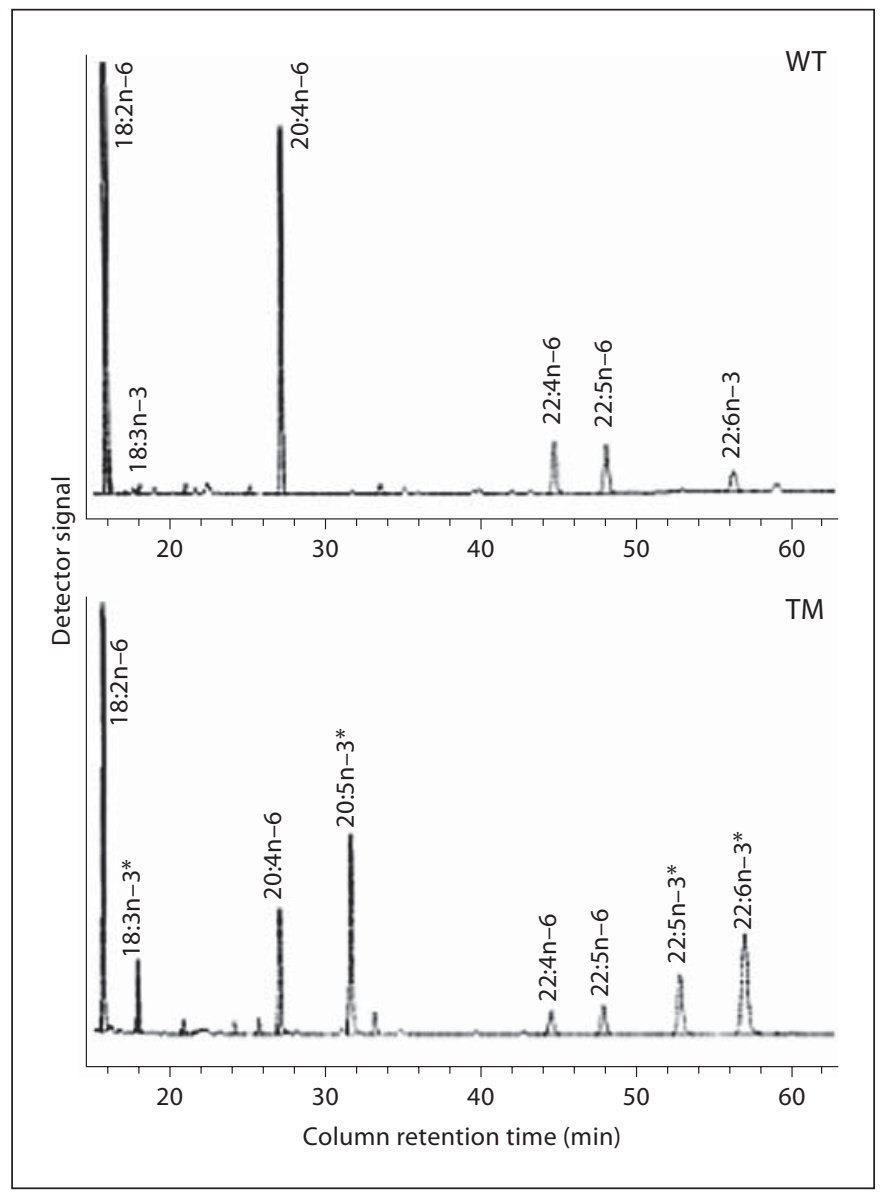

Fig. 2. Partial gas chromatograph traces showing the polyunsaturated fatty acid profiles of total lipids extracted from skeletal muscles of a wild-type mouse (WT, upper panel) and a fat-1 transgenic mouse (TM, lower panel). Both the wild-type and transgenic mice were 8-week-old females and fed on the same diet, which is high in $n-6$ but low in $n-3$ fatty acids. Note, the levels of n-6 fatty acids $(18: 2 n-6,20: 4 n-6,22: 4 n-6$ and 22:5n-6) are remarkably lower, whereas $n-3$ fatty acids $\left(^{*}\right)$ are abundant in the transgenic muscle (lower panel) compared with the wild-type muscle in which there are very little n-3 fatty acids (upper panel).

gans/tissues including muscle and milk [7]. These data clearly show that the transgenic mice expressing the fat- 1 gene are capable of producing $n-3$ fatty acids from $n-6$ fatty acids, resulting in enrichment of $n-3$ fatty acids in their organs/tissues without the need of dietary $n-3$ supply, which is impossible in wild-type mammals. The transgenic mice are normal and healthy. To date, many generations of transgenic mouse lines have been examined and their tissue fatty acid profiles showed consistently high levels of $n-3$ fatty acids, indicating that the transgene is transmittable.
The genetic approach is highly effective in altering the tissue ratio of $n-6$ to $n-3$ fatty acids because it not only increases the absolute amount of $n-3$ fatty acids but also significantly decreases the tissue level of $n-6$ fatty acids, leading to a balanced $n-6 / n-3$ fatty acid ratio in body tissues without changing the mass of tissue fatty acids (i.e. the total amount of $n-6+n-3$ is the same, except for the $\mathrm{n}-6 / \mathrm{n}-3$ ratio in comparison with non-transgenic mice, which conventional dietary supplementation hardly achieves). Thus, this model is ideal for addressing the effects of tissue $n-6 / n-3$ ratio on gene expression in the body.

The use of this fat-1 mouse model allows us to produce two different fatty acid profiles (i.e. high vs. low $n-6 / n-3$ ratio) in a litter of mice born to the same mother by using just a single diet (e.g. a high n-6 diet). Specifically, mating a fat-1 male heterozygote with a wild-type female mouse can produce offspring with two different genotypes: half of them being fat-1 transgenic and other half being wildtype. Then these littermates can simply be fed an identical diet but they exhibit distinct fatty acid profiles (high vs. low $n-6 / n-3$ ratios in wild-type and fat- 1 mice, respectively). Thus, this will eliminate the need of two different diets for a comparative study so that the potential variations in impurities, flavor, caloric and other components (including saturated fatty acids, monounsaturated fatty acids, cholesterol, antioxidants, contaminants and other unidentified substances that may also interact with genes) in the supplemented oils (e.g. fish oil vs. corn oil) as well as other factors derived from dietary supplementation, such as inconsistent preparation and storage conditions of diet, and timing and duration of feeding, can be avoided. Thus, use of this model may provide more reliable and definitive results than dietary manipulation.

In addition, use of the fat- 1 mouse model can save a lot of trouble, time and money that are required for a lengthy feeding (usually for 2-3 months or longer) of different diets in dietary supplementation studies. In transgenic mice, the change in fatty acid composition (conversion of n-6 to n-3) occurs as early as in the embryo stage and lasts throughout life. Thus, with this mouse model it is convenient for one to examine the health effects of $n-3$ fatty acids at different ages or time points.

More attractively, fat- 1 transgenic mouse lines can be used to genetically cross with established disease models (transgenic or knockout animals, such as ob/ob obesity model and ApoE-/- atherosclerosis model) to generate combined (fat-1 plus a diseased gene) models, which make it possible to address the effects of $n-3$ fatty acids 


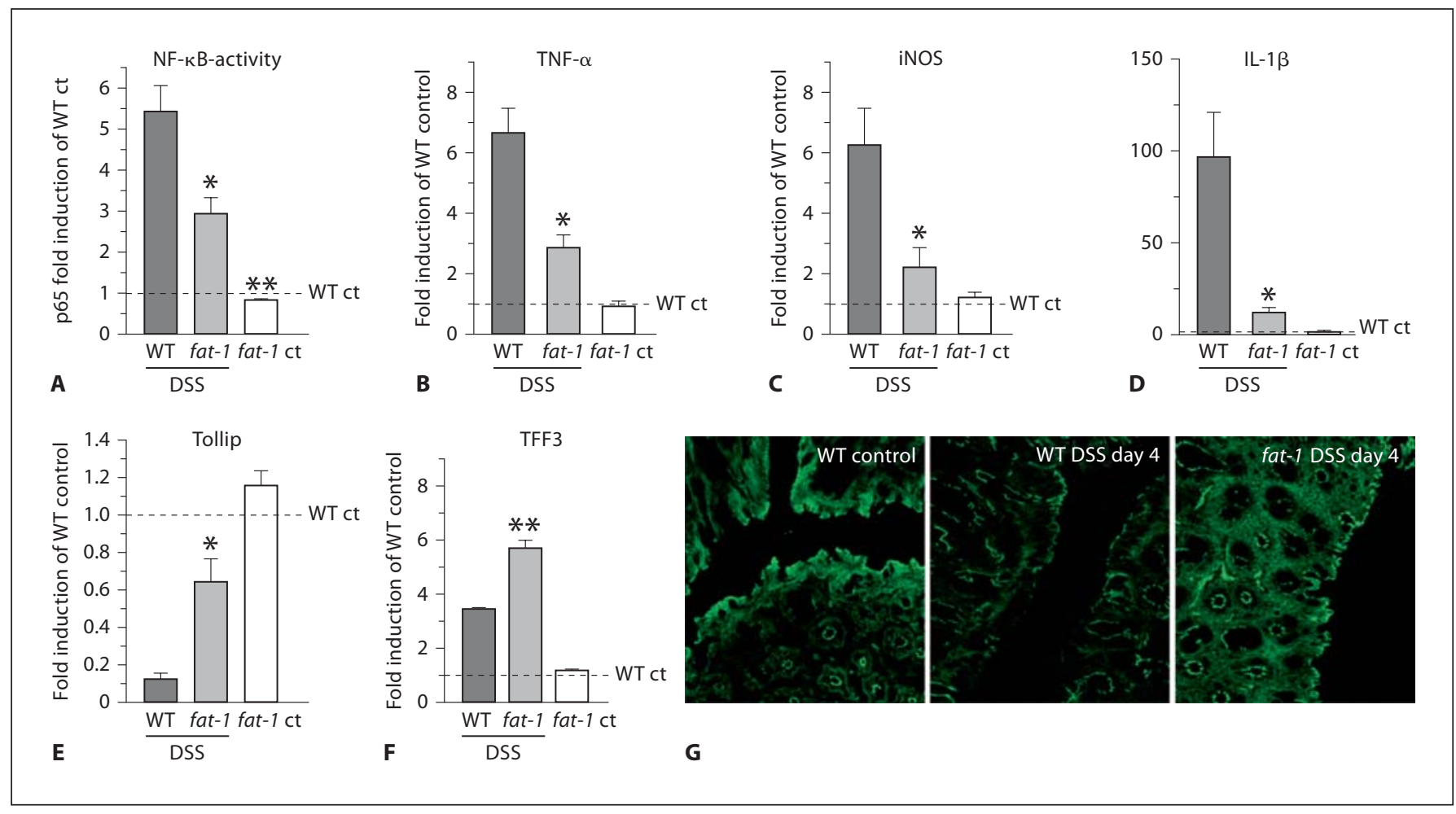

Fig. 3. Gene expression in wild-type (WT) and fat-1 transgenic mice with DSS-induced colitis. A NF- $\kappa \mathrm{B}$ activation, reflected in p65 ELISA activity shows significant differences in control baselines and in disease between WT and fat- 1 mice. ${ }^{*} \mathrm{p}<0.05$ versus WT DSS; ${ }^{* *} \mathrm{p}<0.05$ versus WT control. B-F Semiquantitative real-time PCR analysis of mRNA expression levels of inflammatory mediators TNF $\alpha$, iNOS and IL-1 $\beta$ (B-D) and mucoprotective factors Tollip and TFF3 (E, F) in colons from WT and fat-1 after
DSS-exposure and fat- 1 control mice, normalized as the fold increase to the baseline of WT controls (dashed line). ${ }^{*} \mathrm{p}<0.05$ versus WT DSS; ${ }^{* *} \mathrm{p}<0.01$ versus WT DSS. G ZO-1 expression profile. Compared to wild-type mice without treatment (left panel), $\mathrm{ZO}-1$ expression is downregulated on the luminal epithelial surface in WT mice on day 4 (middle panel), while luminal continuity of expression is sustained in fat-1 mice (right panel).

n-6/n-3 fatty acid ratio on the development of various clinical problems and the expression of related genes [816]. Here I would like to highlight just those published studies related to inflammation and cancer.

\section{Inflammation}

To understand the role played by the tissue status of $\mathrm{n}-3$ fatty acids in the development of inflammatory disease, we utilized fat-1 mice and their counterpart littermates to look at local inflammatory responses, the formation of inflammatory lipid mediators (particularly the newly discovered resolvins and protectins) and the expression of inflammatory genes in the colons following dextran sodium sulfate (DSS) treatment [8]. We found that the inflammation of colon induced by DSS, in terms of both clinical manifestation and pathology, was significantly less severe in fat- 1 transgenic mice than that in 


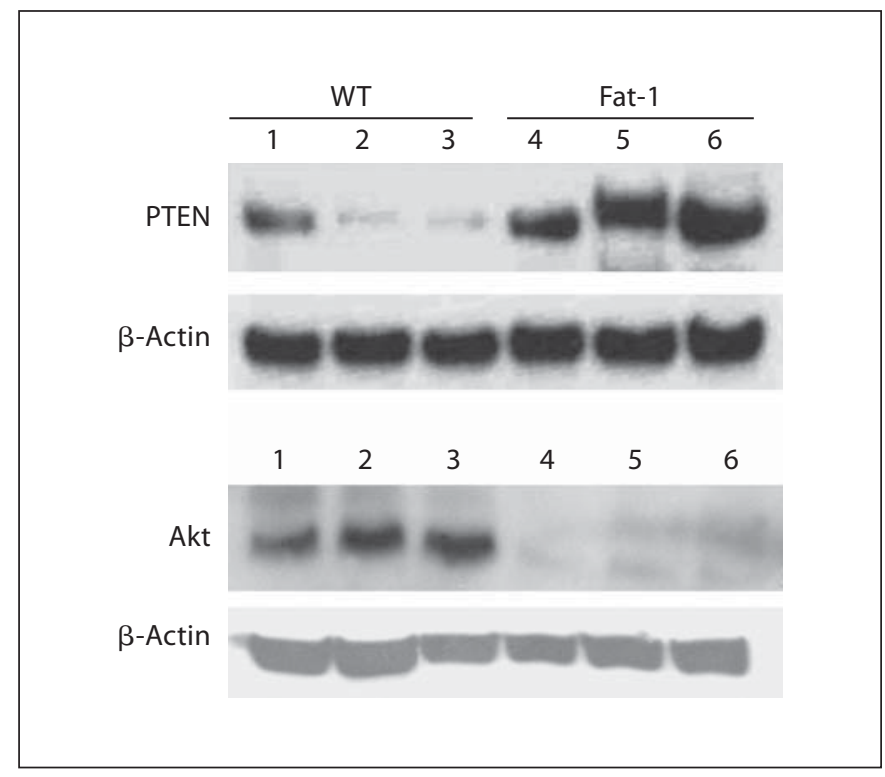

Fig. 4. Western blotting showing differential expression of PTEN and Akt in melanoma tumors from wild-type (WT; lanes 1-3) and fat-1 transgenic (lanes 4-6) mice [11].

their wild-type littermates. The ratio of the long-chain n-6 fatty acid (20:4n-6, 22:4n-6 and 22:5n-6) to the long-chain n-3 fatty acids (20:5n-3, 22:5n-3 and 22:6n3 ) was 1.7 in fat- 1 transgenics and 30.1 in wild-type mice. The protection from colitis in fat-1 mice is correlated with the increased formation of anti-inflammatory derivates of $\mathrm{n}-3$ fatty acids (RvE1 and RvD3, and PD1), downregulation of proinflammatory factors/cytokines (NF- $\mathrm{KB}$, TNF- $\alpha$, iNOS and IL-1 $\beta$; fig. 3 ) and upregulation of mucoprotective factors (TFF3, Tollip and ZO-1; fig. 3) in the colons of these animals [8]. Similarly, in an acute inflammation model (D-GalN/LPS-induced hepatitis) we found less severe inflammatory liver injury in fat- 1 mice, accompanied by reduced hepatic gene expression of the proinflammatory cytokines TNF- $\alpha$, IL- $1 \beta$, IFN- $\gamma$ and IL-6 [9]. In another study, Bhattacharya et al. [10] found that the lower $n-6 / n-3$ ratios in splenocytes from fat- 1 mice on a calorie-restricted diet was associated with significant reductions in the activities of NF- $\mathrm{\kappa B}$ and AP-1, as well as the secretion of IL- 6 and TNF- $\alpha$ following LPS treatment in the splenocytes. Furthermore, Connor et al. [16] used the fat- 1 transgenic mice to examine oxygeninduced retinopathy and found that the protective effect of the increased tissue level of n-3 fatty acids (decreased $n-6 / n-3$ ratio) seen in the fat -1 mice was mediated, in part, through suppression of TNF- $\alpha$. These findings demonstrate the role played by an increased tissue status of $n-3$ fatty acids and decreased $n-6 / n-3$ ratio in protection against inflammation through alterations in gene expression (mainly NF- $\kappa$ B, TNF- $\alpha$, IL- 6 and IL- $1 \beta$ ) mediated, probably, by anti-inflammatory lipid mediators of the $n-3$ fatty acids.

\section{Cancer}

To understand the relationship between the tissue n-6/n-3 fatty acid ratio and tumorigenesis, we implanted mouse melanoma B16 cells into fat-1 and wild-type littermates and examined the incidence of tumor formation and tumor growth rate [11]. The results showed a dramatic reduction in melanoma formation and growth in fat-1 transgenic mice. The levels of $\mathrm{n}-3$ fatty acids and their metabolite $\mathrm{PGE}_{3}$ were much higher (but the $\mathrm{n}-6 /$ $\mathrm{n}-3$ ratio was much lower) in the tumor and surrounding tissues of fat-1 mice than wild-type animals. Accordingly, the PTEN gene (a tumor suppressor) was significantly upregulated in the fat-1 mice (fig. 4). These plus the in vitro data suggest an anti-melanoma effect of $n-3$ fatty acids through, at least in part, activation of the PTEN pathway probably mediated by $\mathrm{PGE}_{3}$ [11]. In another study examining whether increasing colon tissue n-3 fatty acids is effective in preventing colon tumorigenesis, we found that fat- 1 transgenic mice had a lower incidence and growth rate of colon tumors induced by inflammation (DSS) plus treatment with a carcinogen (azoxymethane) [12]. This was accompanied by lower activity of NF$\kappa \mathrm{B}$, higher expression of TGF- $\beta$ in the colons, and lower expression of iNOS in the tumors of fat- 1 animals [12]. Furthermore, to determine the influence of $n-6$ and $n-3$ fatty acids on prostate cancer risk in animals with a defined genetic lesion (Pten deletion), Berquin et al. [13] crossed the fat-1 mice with prostate-specific Pten-knockout mice (a prostate cancer model) to generate compound mice (Pten-knockout plus fat-1). They found that the hybrid mice (Pten-knockout plus fat-1, which have higher levels of n-3 fatty acids) had a significantly lower rate of tumor growth and survived longer when compared with the Pten-knockout mice (without fat-1) [13]. Tumors from mice with high n-3 had lower proportions of phosphorylated Bad, resulting in increased cell apoptosis [13]. They concluded that n-3 and n-6 fatty acids have opposite effects on tumor growth. Similar protective effects of $n-3$ fatty acids in reducing the risk of breast cancer by promoting mammary gland differentiation have also been observed in the fat-1 transgenic mice [14, 15]. In addition, the study of Connor et al. [16] indicates that the reduced pathological angiogenesis in the fat- 1 mice was associated with decreased generation of TNF- $\alpha$. 
Many other studies using fat- 1 transgenic mice are still under way, and so more data related to fatty acid-gene interaction derived from this model will be available soon.

\section{Perspectives}

The fat-1 transgenic mouse is a model of increased tissue content of $n-3$ fatty acids with a balanced $n-6 / n-3$ fatty acid ratio. The use of fat- 1 transgenic mice in the study of n-3 fatty acids can eliminate the need of feeding animals a diet supplemented with $n-3$ fatty acids and thereby avoid the potential confounding effects derived from dietary supplementation. Thus, this mouse model can provide a well-controlled experimental condition (a clean system) for studying the biological effects of $n-3$ fatty acids, and is an important addition to the conventional methods used in this field. Combined use of the fat-1 model with the modern technologies of lipidomics, genetics and proteomics will be a powerful approach to the elucidation of the interactions between the fatty acids or their derivates and genes/proteins. Specifically, tissue samples from transgenic mice and their control littermates can be analyzed for fatty acid and lipid mediator profiles using lipidomic methods (GC-MS and LC-MS/ MS), and simultaneously for gene/protein expression profiles by genetic/proteomic methods (microarray, 2Delectropheresis, etc.). These studies will generate reliable and meaningful results for understanding n-3 fatty acidgene interactions.

\section{Acknowledgements}

This work was supported by grants from the American Cancer Society (RSG-03-140-01-CNE) and the National Institutes of Health (R01CA-113605).

\section{References}

1 Kang JX: From fat to fat-1: a tale of omega-3 fatty acids. J Membr Biol 2005;206:165-172.

2 Leaf A, Weber PC: A new era for science in nutrition. Am J Clin Nutr 1987;45:10481053.

3 Simopoulos AP: Human requirement for $n-$ 3 polyunsaturated fatty acids. Poult Sci 2000 ; 79:961-970.

4 Balk EM, Horsley TA, Newberry SJ, Lichtenstein AH, Yetley EA, Schachter HM, Moher D, MacLean CH, Lau J: A collaborative effort to apply the evidence-based review process to the field of nutrition: challenges, benefits, and lessons learned. Am J Clin Nutr 2007;85: 1448-1456.

5 Spychalla JP, Kinney AJ, Browse J: Identification of an animal omega-3 fatty acid desaturase by heterologous expression in Arabidopsis. Proc Natl Acad Sci USA 1997;94: 1142-1147.

6 Kang ZB, Ge Y, Chen ZH, Brown J, Laposata M, Leaf A, Kang JX: Adenoviral gene transfer of C. elegans n-3 fatty acid desaturase optimizes fatty acid composition in mammalian cells. Proc Natl Acad Sci USA 2001;98: 4050-4054.

7 Kang JX, Wang J, Wu L, Kang ZB: Transgenic mice: fat- 1 mice convert $n-6$ to $n-3$ fatty acids. Nature 2004;427:504.
8 Hudert C, Weylandt KH, Wang J, Lu Y, Song H, Dignass A, Serhan CN, Kang JX: Fat-1 transgenic mice are protected from experimental colitis. Proc Natl Acad Sci USA 2006; 103:11276-11281.

9 Schmöcker C, Weylandt KH, Kahlke L. Wang J, Lobeck H, Tiegs G, Berg T, Kang JX: Omega-3 fatty acids alleviate D-GaIN/LPS induced acute hepatitis by suppression of cytokines. Hepatology 2007;45:864-869.

10 Bhattacharya A, Chandrasekar B, Rahman MM, Banu J, Kang JX, Fernandes G: Inhibition of inflammatory response in transgenic fat-1 mice on a calorie-restricted diet. Biochem Biophys Res Commun 2006;349:925930.

11 Xia SH, Wang J, Lu Y, Song H, Serhan CN, Kang JX: The growth of melanoma is reduced in Fat-1 transgenic mice: Impact of n-6/n-3 essential fatty acids. Proc Natl Acad Sci USA 2006;103:12499-12504.
12 Nowak J, Weylandt KH, Habbel P, Wang J, Dignass A, Glickman JN, Kang JX: Colitisassociated colon tumorigenesis is suppressed in transgenic mice rich in endogenous $n-3$ fatty acids. Carcinogenesis 2007;28:19911995.

13 Berquin IM, Min Y, Wu R, Wu J, Perry D, Cline JM, Thomas MJ, Thornberg T, Smith A, Edwards IJ, D’Agostino R, Zhang H, Wu H, Kang JX, Chen YQ: Modulation of prostate cancer genetic risk by omega-3 and omega-6 fatty acids. J Clin Invest 2007;117: 1866-1875.

14 Ma DWL, Ngo V, Huot P, Kang JX: Omega-3 polyunsaturated fatty acids endogenously synthesized in fat-1 mice are enriched in the mammary gland. Lipids 2006;41:35-39.

15 Liu YE, Pu W, Wang J, Kang JX, Shi YE: Activation of Stat 5 and induction of a pregnancy-like mammary gland differentiation by eicosapentaenoic and docosapentaenoic omega-3 fatty acids. FEBS J 2007:274;33513362 .

16 Connor KM, SanGiovanni JP, Lofqvist C, Aderman CM, Chen J, Higuchi A, Hong S, Pravda E, Majchrzak S, Carper D, Hellstrom A, Kang JX, Chew EY, Salem N, Serhan CN, Smith LEH: Increasing dietary intake of $\omega-3$ PUFA reduces pathological retinal angiogenesis. Nat Med 2007;13:868-873. 